\title{
BOOTES Observation of GRB 080603B
}

\author{
M. Jelínek, J. Gorosabel, A. J. Castro-Tirado, A. de Ugarte Postigo, S. Guziy, R. Cunniffe, \\ P. Kubánek, M. Prouza, S. Vítek, R. Hudec, V. Reglero, L. Sabau-Graziati
}

\begin{abstract}
We report on multicolor photometry of long GRB 080603B afterglow from BOOTES-1B and BOOTES-2. The optical afterglow has already been reported to present a break in the optical lightcurve at $0.12 \pm 0.2$ days after the trigger. We construct the lightcurve and the spectral energy distribution and discuss the nature of the afterglow.
\end{abstract}

Keywords: gamma-ray bursts, individual, GRB 080603B.

\section{Introduction}

GRB 080603B was a long gamma-ray burst detected on June 3, 2008, at 19:38:13 UT by Swift-BAT [14]. The burst was also detected by Konus-WIND [5] and INTEGRAL-SPI/ACS [18].

In X-rays, the afterglow was detected by SwiftXRT, providing a rapid and precise localization [13].

The optical afterglow was observed by several telescopes - ROTSE III [19], TAROT [9-11], TLS Tautenburg [8], RTT150 [24], the Liverpool Telescope [15], Xinglong EST [23], the $1.0 \mathrm{~m}$ telescope at $\mathrm{CrAO}[21,20]$, the $1.5 \mathrm{~m}$ telescope of Sayan observatory [12] and from Maidanak [6]. In infrared by PAIRITEL [16], spectroscopy was obtained by the NOT [4] and the Hobby-Eberly Telescope [2], providing a redshift of $z=2.69$.

An upper limit on radio emission was set by the VLA [1].

\section{Observations}

At both BOOTES stations, the GRB happened during twilight, delaying follow-up by $\sim 1 \mathrm{~h}$. Despite the delay, the optical afterglow is well detected in the data from both telescopes.

The $60 \mathrm{~cm}$ telescope BOOTES-2/TELMA, in La Mayora, Málaga, Spain, started taking data at 20:29:19 UT, i.e. 51 minutes after the GRB trigger. A sequence of $r^{\prime}$-band exposures was taken, and later, after confirming the detection of the optical transient, $i^{\prime}, g^{\prime}$ and $\mathrm{Y}$ band images were obtained. In the near infrared Y band, despite $600 \mathrm{~s}$ of integration, the afterglow was not detected.

The $30 \mathrm{~cm}$ telescope BOOTES-1B, located in El Arenosillo, Huelva, Spain, [7] obtained 368 unfiltered images totalling more than 6 hours of integrated light until the end of the night. The images were combined to improve signal-to-noise, to yield 11 data points for the period between 1.2 and 5.2 hours after the GRB.
One point has a large error due to clouds crossing the field of view.

The best fit astrometric position of the afterglow, obtained from the weighted average of all available images from BOOTES-2 is

$$
\alpha=11: 46: 07.73 \quad \delta=+68: 03: 39.9 \quad(J 2000),
$$

about $1.6^{\circ} \mathrm{SE}$ from star $\lambda$ Dra.

Photometry was done in the optimal aperture using IRAF/Daophot. Calibration was performed against three SDSS (DR8) [3] stars. The stars are marked on the identification chart (Figure 3) and their brightnesses are in the Table 1. Our unfiltered, "Clear", best fit magnitude Clear $=A_{1} * g^{\prime}+A_{2} * r^{\prime}$ used for BOOTES-1B calibration is also mentioned.

For the summary of our observations, see Table 2.

Table 1: Calibration stars used

\begin{tabular}{ccccc}
\hline ID. & $g^{\prime}$ & $r^{\prime}$ & $i^{\prime}$ & Clear \\
\hline 1 & 18.00 & 17.50 & 17.32 & 17.52 \\
2 & 18.80 & 17.35 & 16.04 & 17.35 \\
3 & 19.88 & 18.42 & 17.09 & 18.47 \\
\hline
\end{tabular}

\section{$3 \quad$ Fitting the lightcurve}

The lightcurve, as already shown by [24] shows a smooth transition between two decay slopes $\alpha_{1}=$ $-0.55 \pm 0.16$ and $\alpha_{2}=-1.23 \pm 0.22$. The break occurs at $t_{b}=0.129 \pm 0.016$ days.

There is no hint of chromatic evolution within the lightcurve, so all filters were scaled and fitted together with the $r^{\prime}$-band. The fitting of the lightcurve was performed in $\log t / \log f$ space, where power law functions, typical for gamma-ray bursts, show as straight lines. We used a hyperbolic transition between two slopes (smoothly broken power-law): 
Table 2: Optical photometric observations of the optical afterglow of the GRB 080603B

\begin{tabular}{|c|c|c|c|c|c|c|}
\hline UT Date of mid exp. & $T-T_{0}[\mathrm{~h}]$ & tel. & filter & $T_{\exp }[\mathrm{s}]$ & mag & $\delta \mathrm{mag}$ \\
\hline Jun 3.855805 & 0.902 & B-2 & $r^{\prime}$ & $3 \times 120 \mathrm{~s}$ & 17.46 & 0.07 \\
\hline Jun 3.859348 & 0.987 & B-2 & $r^{\prime}$ & $2 \times 120 \mathrm{~s}$ & 17.59 & 0.13 \\
\hline Jun 3.862188 & 1.056 & B-2 & $r^{\prime}$ & $2 \times 120 \mathrm{~s}$ & 17.31 & 0.05 \\
\hline Jun 3.864311 & 1.107 & B-2 & $r^{\prime}$ & $120 \mathrm{~s}$ & 17.57 & 0.08 \\
\hline Jun 3.865747 & 1.141 & B-2 & $r^{\prime}$ & $120 \mathrm{~s}$ & 17.30 & 0.07 \\
\hline Jun 3.867151 & 1.175 & B-2 & $r^{\prime}$ & $120 \mathrm{~s}$ & 17.46 & 0.06 \\
\hline Jun 3.868946 & 1.218 & B-1B & Clear & $10 \times 60 \mathrm{~s}$ & 17.53 & 0.07 \\
\hline Jun 3.870011 & 1.243 & B-2 & $g^{\prime}$ & $3 \times 120 \mathrm{~s}$ & 18.29 & 0.04 \\
\hline Jun 3.874248 & 1.345 & B-2 & $g^{\prime}$ & $3 \times 120 \mathrm{~s}$ & 18.24 & 0.04 \\
\hline Jun 3.876758 & 1.405 & B-1B & Clear & $10 \times 60 \mathrm{~s}$ & 17.54 & 0.06 \\
\hline Jun 3.879225 & 1.465 & B-2 & $g^{\prime}$ & $4 \times 120 \mathrm{~s}$ & 18.14 & 0.03 \\
\hline Jun 3.884248 & 1.585 & B-2 & $r^{\prime}$ & $3 \times 120 \mathrm{~s}$ & 17.50 & 0.09 \\
\hline Jun 3.884664 & 1.595 & B-1B & Clear & $10 \times 60 \mathrm{~s}$ & 17.70 & 0.06 \\
\hline Jun 3.889912 & 1.721 & B-2 & $r^{\prime}$ & $3 \times 120 \mathrm{~s}$ & 17.70 & 0.15 \\
\hline Jun 3.892654 & 1.787 & B-1B & Clear & $10 \times 60 \mathrm{~s}$ & 17.75 & 0.06 \\
\hline Jun 3.893455 & 1.806 & B-2 & $r^{\prime}$ & $4 \times 120 \mathrm{~s}$ & 17.74 & 0.06 \\
\hline Jun 3.899839 & 1.959 & B-2 & $g^{\prime}$ & $5 \times 120 \mathrm{~s}$ & 18.42 & 0.19 \\
\hline Jun 3.900620 & 1.978 & B-1B & Clear & $10 \times 60 \mathrm{~s}$ & 17.79 & 0.06 \\
\hline Jun 3.906961 & 2.130 & B-2 & $g^{\prime}$ & $5 \times 120 \mathrm{~s}$ & 18.42 & 0.04 \\
\hline Jun 3.908509 & 2.167 & B-1B & Clear & $10 \times 60 \mathrm{~s}$ & 17.87 & 0.09 \\
\hline Jun 3.914867 & 2.320 & B-2 & $r^{\prime}$ & $4 \times 120 \mathrm{~s}$ & 18.15 & 0.13 \\
\hline Jun 3.916482 & 2.359 & B-1B & Clear & $10 \times 60 \mathrm{~s}$ & 17.91 & 0.11 \\
\hline Jun 3.922694 & 2.508 & B-2 & $i^{\prime}$ & $5 \times 120 \mathrm{~s}$ & 17.89 & 0.05 \\
\hline Jun 3.931774 & 2.726 & B-2 & $r^{\prime}$ & $7 \times 120 \mathrm{~s}$ & 18.01 & 0.06 \\
\hline Jun 3.934988 & 2.803 & B-1B & Clear & $35 \times 60 \mathrm{~s}$ & 18.30 & 0.32 \\
\hline Jun 3.940845 & 2.943 & B-2 & $i^{\prime}$ & $5 \times 120 \mathrm{~s}$ & 17.88 & 0.07 \\
\hline Jun 3.947882 & 3.112 & B-2 & $r^{\prime}$ & $5 \times 120 \mathrm{~s}$ & 18.12 & 0.08 \\
\hline Jun 3.956941 & 3.330 & B-1B & Clear & $20 \times 60 \mathrm{~s}$ & 18.45 & 0.07 \\
\hline Jun 3.971736 & 3.685 & B-1B & Clear & $21 \times 60 \mathrm{~s}$ & 18.38 & 0.06 \\
\hline Jun 3.977109 & 3.814 & B-2 & $r^{\prime}$ & $5 \times 120 \mathrm{~s}$ & 18.26 & 0.18 \\
\hline Jun 4.006997 & 4.531 & B-1B & Clear & $78 \times 60 \mathrm{~s}$ & 18.79 & 0.07 \\
\hline
\end{tabular}

$$
h(a, b)=a+\frac{b}{2} \sqrt{1+\frac{a^{2}}{b^{2}}}
$$

$m(t)=m_{0}-2.5 \alpha_{2} \log \frac{t}{t_{b}}+h\left(-2.5\left(\alpha_{1}-\alpha_{2}\right) \log \frac{t}{t_{b}}, G\right)$

where $\alpha_{1}$ and $\alpha_{2}$ are pre-break and post-break decay indices, $t_{b}$ is the break time, $m_{0}$ is an absolute scaling parameter of the brightness and $G$ expresses smoothness of the break.

Although the early point by ROTSE [19] was not used, it agrees with the backward extrapolation of the $\alpha_{1}$ slope and so supports this simple interpretation.
We constructed a spectral energy distribution (SED) by fitting the needed magnitude shift of the R-band lightcurve model to the photometric points from BOOTES, UVOT [14] and PAIRITEL [16] obtained in other filters. While the points from UVOT are practically contemporaneous to BOOTES, PAIRITEL observed rather later (0.32 days after the trigger), so the SED is therefore model-dependent in its infrared part. The synthetic $\mathrm{AB}$ magnitudes equivalent to $t=0.1$ days are in Table 3 . 


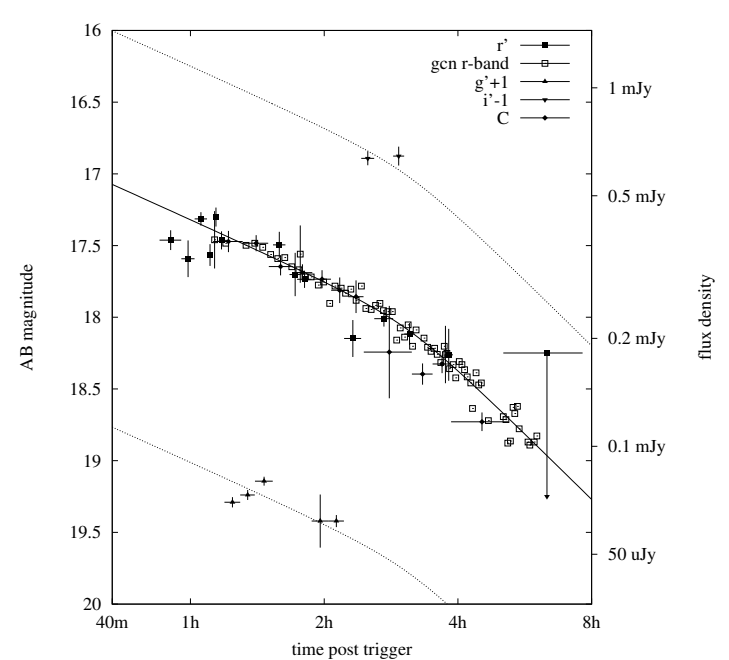

Fig. 1: Detail of the optical light curve of GRB 080603B showing the observations by BOOTES (filled symbols) and from the literature (empty symbols)

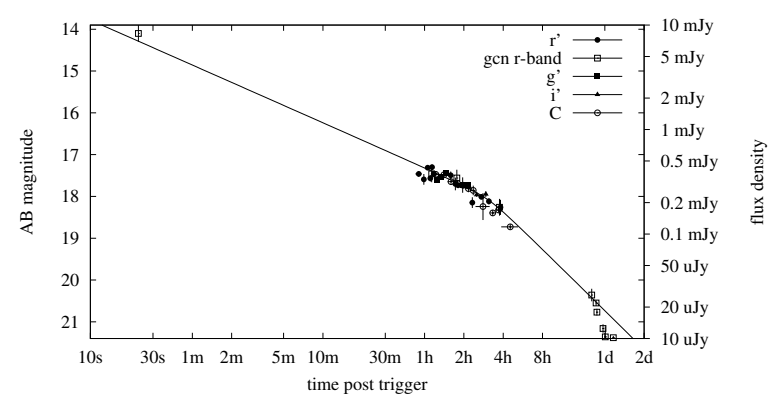

Fig. 2: Overall view of the light curve of GRB 080603B

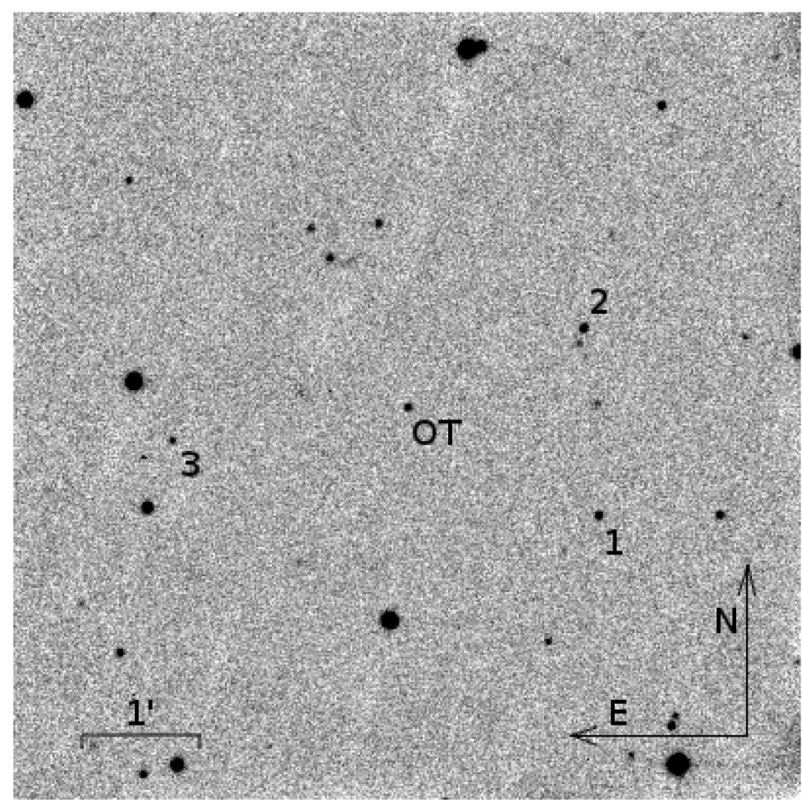

Fig. 3: The finding chart of the afterglow of GRB080603B. Combination of images taken by BOOTES-2

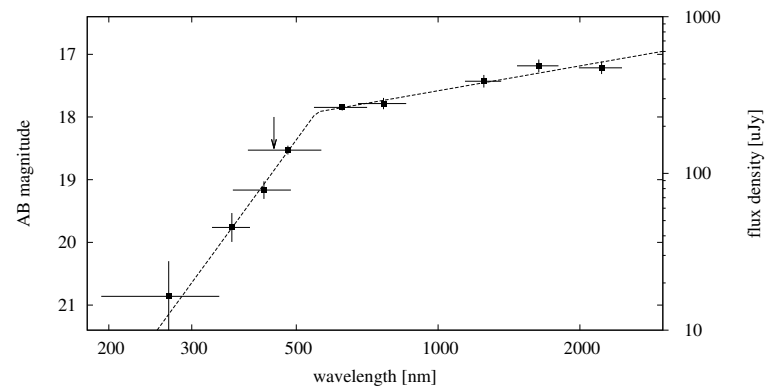

Fig. 4: The spectral energy distribution of the afterglow in rest frame. The small arrow marks Ly- $\alpha$ position for $z=2.69$

Table 3: The spectral energy distribution in AB magnitudes equivalent to 0.1 days after the trigger. $\left({ }^{\dagger} \mathrm{UVOT}\right.$, $\ddagger$ PAIRITEL)

\begin{tabular}{ccc}
\hline Filter & $m_{\mathrm{AB}}$ & $\Delta m_{\mathrm{AB}}$ \\
\hline $\mathrm{W}^{\dagger}$ & 20.98 & 0.56 \\
$\mathrm{U}^{\dagger}$ & 19.83 & 0.23 \\
$\mathrm{~B}^{\dagger}$ & 19.22 & 0.14 \\
$g^{\prime}$ & 18.57 & 0.07 \\
$r^{\prime}$ & 17.88 & 0.05 \\
$i^{\prime}$ & 17.81 & 0.09 \\
$\mathrm{~J}^{\ddagger}$ & 17.44 & 0.10 \\
$\mathrm{H}^{\ddagger}$ & 17.19 & 0.10 \\
$\mathrm{~K}^{\ddagger}$ & 17.22 & 0.10 \\
\hline
\end{tabular}

The SED shows a clear suppression of radiation above $4500 \AA$, i.e. a redshifted Ly- $\alpha$ line. No radiation is detected above the Lyman break at $3365 \AA$. A rather shallow power law with an index $\beta=-0.53 \pm$ 0.06 was found redwards from $r^{\prime}$ band. The fit was performed using the $E(B-V)=0.013 \mathrm{mag}[22]$.

The strong suppression of light for wavelengths shorter than $r^{\prime}$ band is likely due to the Ly- $\alpha$ absorption within the host galaxy and Ly-alpha line blanketing for $z=2.69$.

\section{Discussion}

The values of $\alpha_{2}=-1.23 \pm 0.22$ and $\beta=-0.53 \pm 0.06$ both point to a common electron distribution parameter $p=2.05 \pm 0.20(\alpha=(3 * p-1) / 4, \beta=$ $(p-1) / 2)[17]$. Such a combination suggests a stellar wind profile expansion and a slow cooling regime.

The pre-break decay rate $\alpha_{1}=-0.55 \pm 0.16$ remains unexplained by the standard fireball model. It is unlikely that the break at $t_{b}=0.129 \pm 0.016$ would be a jet break. It is quite possible that the plateau is not really a straight power law, and that some late 
activity of the inner engine may be producing bumping of hydrodynamic origin.

We note that the literature contains a number of observations suggesting a rapid decay by about one day after the GRB. Without having all the images, it is, however, impossible to decide whether this is a real physical effect or a zero-point mismatch.

\section{Conclusions}

The $0.6 \mathrm{~m}$ telescope BOOTES-2 in La Mayora observed the optical afterglow of GRB 080603B in three filters. The $0.3 \mathrm{~m}$ BOOTES-1B in El Arenosillo observed the same optical afterglow without a filter.

Using the data we obtained at BOOTES and from the literature, we construct the lightcurve and broadband spectral energy distribution.

Our fit of the obained data privides the decay parameters $\alpha_{2}=1.23 \pm 0.22$ and $\beta=-0.53 \pm 0.06$, which suggest a slow cooling expansion into a stellar wind.

\section{Acknowledgement}

We acknowledge the support of the Spanish Ministerio de Ciencia y Tecnología through Projects AYA2008-03467/ESP and AYA2009-14000-C03-01/ ESP, and Junta de Andalucía through the Excellence Reseach Project P06-FQM-219, and the GAČR grants 205/08/1207 and 102/09/0997. We are also indebted to T. Mateo-Sanguino (UHU), J. A. Adame, J. A. Andreu, B. de la Morena, J. Torres (INTA) and to R. Fernández-Munoz (EELM-CSIC), V. MunozFernández and C. Pérez del Pulgar (UMA) for their support.

\section{References}

[1] Chandra, P., Frail, D. A.: GRB 080603B: PAIRITEL infrared detection. GCN Circular, 7827, 2008.

[2] Cucchiara, A., Fox, D.: GRB 080603B: HobbyEberly Telescope redshift confirmation. GCN Circular, 7 815, 2008.

[3] Eisenstein, D. J., Weinberg, D. H., Agol, E., Aihara, H., Allende Prieto, C., Anderson, S. F., Arns, J. A., Aubourg, É., Bailey, S., Balbinot, E., et al.: SDSS-III: Massive Spectroscopic Surveys of the Distant Universe, the Milky Way, and Extra-Solar Planetary Systems. $A J$, 142, 72, Sept. 2011.

[4] Fynbo, J., Quirion, P.-O., Xu, D., Malesani, D., Thoene, C., Hjorth, J., Milvang-Jensen, B., Jakobson, P.: GRB 080603B: NOT redshift. GCN Circular, 7 797, 2008.
[5] Golenetskii, S., Aptekar, R., Mazets, E., Pal'shin, V., Frederiks, D., Cline, T.: KonusWind observation of GRB 080603B. GCN Circular, 7 812, 2008.

[6] Ibrahimov, M., Karimov, P., Rumyantsev, A., Pozanenko, A.: GRB 080603B: optical observations in MAO. GCN Circular, 7 975, 2008.

[7] Jelínek, M., Castro-Tirado, A. J., de Ugarte Postigo, A., Kubánek, P., Guziy, S., Gorosabel, J., Cunniffe, R. Vítek, S., Hudec, R., Reglero, V., Sabau-Graziati, L.: Four Years of Real-Time GRB Followup by BOOTES-1B (2005-2008). Advances in Astronomy, 2 010, $432172,2010$.

[8] Kann, D., Laux, U., Ertel, S.: GRB 080603B: TLS Afterglow Observation. GCN Circular, $7 \mathbf{8 2 3}, 2008$.

[9] Klotz, A., Boer, M., Atteia, J.: GRB 080603B: TAROT Calern observatory detection of a plateau in the light curve. GCN Circular, $7 \mathbf{7 9 5}$, 2008.

[10] Klotz, A., Boer, M., Atteia, J.: GRB 080603B: TAROT Calern observatory confirmation of slow optical decay. GCN Circular, 7 799, 2008.

[11] Klotz, A., Boër, M., Atteia, J., Gendre, B.: Early Optical Observations of Gamma-Ray Bursts by the TAROT Telescopes: Period 2001-2008. The Astronomical Journal, 2009.

[12] Klunko, E., Pozanenko, A.: GRB 080603B: optical observation. GCN Circular, 7 890, 2008.

[13] Mangano, V., La Parola, B., Sbarufatti, B.: GRB 080603B: Swift-XRT refined analysis. GCN Circular, 7 806, 2008.

[14] Mangano, V., Parsons, A., Sakamoto, T., La Parola, V., Kuin, N., Barthelmy, S., Burrows, D., Roming, P., Gehrels, N.: Swift Observation of GRB 080603B. GCN Report, 144, 2008.

[15] Melandri, A., Gomboc, A., Guidorzi, C., Smith, R., Steele, I., Bersier, D., Mundell, C., Carter, D., Kobayashi, S., Burgdorf, M., Bode, M., Rol, E., O'Brien, P., Bannister, N., Tanvir, N.: GRB 080603B: Liverpool Telescope Observations. GCN Circular, 7 813, 2008.

[16] Miller, A., Bloom, J., Perley, D.: GRB 080603B: PAIRITEL infrared detection. GCN Circular, $7827,2008$.

[17] Piran, T.: The physics of gamma-ray bursts. Reviews of Modern Physics, 76, 1143-1210, Oct. 2004. 
[18] Rau, A.: Catalogue of SPI-ACS Gamma-Ray Burst. http://www.mpe.mpg.de/gamma/science/ grb/1ACSburst.html, 2012.

[19] Rujopakarn, W., Guver, T., Smith, D.: GRB 080603B: ROTSE-III Detection of Optical Counterpart. GCN Circular, 7 792, 2008.

[20] Rumyantsev, A., Antoniuk, K., Pozanenko, A.: GRB 080603B: optical observations in CrAO. GCN Circular, 7 974, 2008.

[21] Rumyantsev, V., Pozanenko, A.: GRB 080603B: optical observation. GCN Circular, $\mathbf{7} \mathbf{8 6 9}, 2008$.

[22] Schlegel, D., Finkbeiner, D., Davis, M.: Maps of Dust Infrared Emission for Use in Estimation of Reddening and Cosmic Microwave Background Radiation Foregrounds. AJP, 500, 525, June 1998.

[23] Xin, L., Feng, Q., Zhai, M., Qiu, Y., Wei, J., Hu, J., Deng, J., Wang, J., Urata, Y., Zheng, W.: GRB 080603B: Xinglong EST observations. GCN Circular, $\mathbf{7} \mathbf{8 1 4}, 2008$.

[24] Zhuchkov, R., Bikmaev, I., Sakhibullin, N., Khamitov, I., Eker, Z., Kiziloglu, U., Gogus, E., Burenin, R., Pavlinsky, M., Sunyaev, R.: GRB 080603B: RTT150 optical observations, break in light curves. GCN Circular, $\mathbf{7 8 0 3}$, 2008 .
Martin Jelínek

E-mail: mates@iaa.es

Instituto de Astrofísica de Andalucía CSIC

Granada, Spain

Javier Gorosabel

Alberto J. Castro-Tirado

Antonio de Ugarte Postigo

Sergei Guziy

Ronan Cunniffe

Instituto de Astrofísica de Andalucía CSIC

Granada, Spain

Petr Kubánek

Michael Prouza

Fyzikální ústav (FzÚ AV ČR)

Praha, Czech Republic

Stanislav Vítek

Fakulta Elektrotechnická, ČVUT v Praze, Czech Republic

René Hudec

Astronomický ústav Akademie věd (ASÚ AV ČR)

Ondřejov, Czech Republic

Fakulta Elektrotechnická, ČVUT v Praze, Czech Republic

Victor Reglero

Image Processing Laboratory

Universitat de Valencia, Spain

Lola Sabau-Graziati

Instituto Nacional de Técnica Aeroespacial

Torrejón de Ardoz, Madrid, Spain 